\section{Le bureau d'expertises et la révision de son règlement}

Médecin et patient forment une communauté d'intérêts fondée sur la confiance. Une confiance qui a tendance à se lézarder, si le succès thérapeutique se fait attendre ou si un dommage s'y ajoute, même. Une faute présumée par le patient, concernant le diagnostic ou le traitement, voire les deux, peut rendre difficile la communication entre médecin et patient. Cette situation peut être évitée en soumettant, dans l'intérêt bien compris des parties, les faits médicaux allégués à une expertise indépendante.

Le Bureau d'expertises extra-judiciaires de la FMH se consacre depuis 1982 à cette noble et complexe tâche servant également à l'assurance-qualité de l'assistance médicale. Il saisit l'occasion de ses vingt années d'existence et acquise avec quelque 2500 cas traités pour remanier son règlement et les documents y attenant.

L'objectif ultime reste inchangé: trouver la vérité et obtenir une appréciation médicale permettant de régler l'affaire à l'amiable et, dans la foulée, de faire profiter des enseignements tirés la formation des médecins. Les violations des règles reconnues de la médecine classique sont à indemniser équitablement sur le plan du droit en matière de responsabilité civile. Seules les complications inévitables et fatidiques, quelle que soit la diligence apportée, doivent rester sans suites judiciaires.

En ma qualité de représentant des intérêts des patients dans les cas de responsabilité civile des hôpitaux et des médecins, j'adhère sans réserve à cette ligne de conduite. Ces questions me préoccupent depuis 25 ans et de l'eau a passé sous les ponts depuis ma thèse, présentée en 1978, sur les "Voraussetzungen der zivilrechtlichen Haftung des Arztes" ["conditions de la responsabilité civile $d u$ médecin"; note du trad.]. La pratique judiciaire et extrajudiciaire nous montre que si l'on est en droit, dans notre pays, d'exiger du médecin de vastes connaissances et un sens aigu des responsabilités, on ne saurait néanmoins attendre de lui qu'il garantisse le succès sans réserve de ses actes médicaux. Nous ne saurions, ni ne voudrions introduire chez nous des règles à l'américaine. Telle est la "bonne» nouvelle. La «mauvaise», si l'on peut dire, c'est que les patients ont acquis une conscience plus aiguë de leurs droits en cas de dommage. Droit de protection des consommateurs aidant, ils recourent aujourd'hui plus souvent à la sauvegarde de leurs droits. Il est dès lors d'autant plus important de renforcer, pour les dommages, la collaboration constructive et efficace entre, d'une part, les médecins, les assureurs, les organisations de patients et les avocats spécialisés et, d'autre part, le Bureau d'expertises extrajudiciaires de la FMH, les sociétés de discipline médicale et les experts. La tâche de ces derniers est la plus importante. L'expertise de la FMH doit répondre de manière objective, neutre et sans équivoque (en langage clair) aux questions médicales concernant les faits. Il s'agit de mettre en lumière la question de la faute professionnelle éventuelle et la présence du rapport naturel de causalité, la question de l'appréciation juridique $d u$ cas d'espèce demeurant du ressort des juristes. Le problème est que les juristes et les médecins ne parlent pas toujours le même langage, ce qui ne facilite pas la compréhension de part et d'autre. Il faut dès lors que toutes les conclusions de l'expert soient établies, confirmées par la littérature scientifique du moment, accessibles au béotien et surtout vérifiables.

Il convient de mettre fin définitivement à un vieux malentendu: le litige en matière de responsabilité civile n'a jamais pour objet d'attaquer le médecin personnellement ou de le dénigrer sur le plan professionnel. Il s'agit uniquement d'aider à réaliser le souhait exprimé au début de cet exposé. Cela dit, le patient est placé devant la difficile situation de devoir fournir les preuves de ce qu'il avance, dans toute la subjectivité de ses impressions personnelles de profane. D'autre part, l'expert médecin détient le savoir spécifique, connaît le dossier du patient, peut consulter les résultats objectifs, bref, il dispose de quelques longueurs d'avance en termes de connaissances médicales. Tout médecin en butte à des reproches de patients ressentis comme injustifiés, devrait en être conscient.

Dans ce champ clos d'intérêts contradictoires, le Bureau d'expertises extrajudiciaires de la FMH tient un rôle important; il ne pourra que profiter de la révision en cours. Il me reste à lui souhaiter plein succès pour les deux prochaines décennies.

Me Werner E. Ott Avocat, Zurich 